\title{
Inhomogeneous space-times admitting isotropic radiation: Vorticity-free case
}

\author{
Joan Josep Ferrando, Juan Antonio Morales, and Miquel Portilla \\ Departament de Física Teòrica, Universitat de Valencia, 46100 Burjassot, Valencia, Spain
}

(Received 5 December 1991)

\begin{abstract}
The energy-momentum tensor of space-times admitting a vorticity-free and a shear-free timelike congruence is obtained. This result is used to write Einstein equations in a convenient way in order to get inhomogeneous space-times admitting an isotropic distribution of photons satisfying the Liouville equation. Two special cases with anisotropic pressures in the energy flow direction are considered.
\end{abstract}

PACS number(s): 04.20.Jb

\section{INTRODUCTION}

The highly isotropic microwave background (interpreted as a relic of an early hot stage of the Universe) is considered as proof that the space-time, on a sufficiently large scale, is close to a Robertson-Walker universe. This has been stated in a paper by Ehlers, Geren, and Sachs [1] (EGS) and summarized in the book by Hawking and Ellis [2], who also proved that space-time should be timelike or null geodesically incomplete before the decoupling of matter and radiation, supporting in this way the bigbang models. On the other hand, small deviations from homogeneity at decoupling time are necessary in order to understand the formation of the observed structures; and, after the work by Sachs and Wolfe [3], a pattern of anisotropies in the microwave background (MWB) temperature is predicted.

Let us dwell a little more on this basic subject, recalling the assumptions necessary to arrive at these conclusions. The EGS theorem starts from previous results $[4,1]$ characterizing the class of space-times allowing isotropic radiation: the metric should be conformally stationary and the distribution function depends only on the first integral of the null geodesic equation, defined by the conformal Killing vector. In fact this is all one can obtain from the existence of a collisionless isotropic distribution of photons. The result of EGS (see Corollary 2 below) for the relation of isotropic radiation to a Robertson-Walker universe arises when (i) the observer who sees an isotropic MWB is geodesic, and (ii) the energy tensor is a perfect fluid comoving with this observer. However, conditions (i) and (ii) should be considered as good approximations to reality, not as exact prescriptions. So, we can modify one or both of them in order to get perturbed Robertson-Walker universes compatible with the isotropy of the MWB. All we have to do is to assume a conformally stationary metric from the beginning. Cosmology is not devoid of examples of these metrics. For instance, the metric used to obtain the Sachs-Wolfe effect belongs to this type. The question arising here is why Sachs and Wolfe established a welldefined relationship between inhomogeneities and anisotropies in the MWB temperature. We have considered this subject elsewhere [5]. Other examples may be found among the Stephani universes $[6,7]$.
In this paper we develop a framework suited to obtain more general inhomogeneous solutions of Einstein equations compatible with isotropic radiation. For the sake of simplicity, we shall assume that the observer measuring isotropic radiation admits orthogonal hypersurfaces. Under these conditions, space-time allows a shear-free and vorticity-free timelike congruence, or equivalently, an umbilical synchronization. Conformally flat umbilical synchronizations exist in any space-time admitting natural symmetric frames [8]. This occurs for any spherically symmetric space-time [9] and also for the Stephani universes [10] that include the Robertson-Walker metrics. On the other hand, dealing with scalar perturbations of Robertson-Walker universes in the longitudinal gauge, one removes the background space-time symmetries but not the existence of an umbilical synchronization [11]. Moreover, after a study made by Treciokas and Ellis [12], the shear-free and vorticity-free timelike congruences constitute a large class among the observers measuring an isotropic distribution function obeying the Boltzmann equation. The study of space-times allowing an umbilical synchronization is justified by this last result as well as by the existence of a lot of usual space-times with this property. The particular case where the observer is geodesic has been previously considered [13].

In Sec. II, using the $3+1$ formalism, we write in a suitable form the Einstein equations for the space-times admitting an umbilical synchronization. The four boundary equations and the six evolution equations become nine boundary equations (with the energy density, the energy flow, and the anisotropic pressures tensor on the initial slice as sources) and a unique second-order evolution equation (with the mean pressure as source).

In Sec. III we summarize the known results of Ehlers, Geren, and Sachs in order to justify the canonic form of the metrics compatible with isotropic radiation. The results obtained in previous sections are used to write Einstein equations when the observer measuring isotropic radiation is hypersurface orthogonal.

In general, inhomogeneous metrics compatible with isotropic radiation will correspond to energy tensors with anisotropic pressures. Section IV is engaged in the study of two particular cases with the pressure tensor presenting anisotropies only in the energy flow direction, as occurs in spherically symmetric space-times. Thus, in a 
sense, solutions with such an energy distribution may be considered as a natural generalization of the solutions with spherical symmetry. Anisotropic pressures of this type have been used in galaxy models [14] and cannot be ruled out by observations of galaxy clusters [15]. Elsewhere [16] we have given the conditions which allow these energy distributions to be interpreted either as a mixture of two noncomoving perfect fluids or as a tilted perfect fluid.

\section{UMBILICAL FOLIATIONS ON SPACE-TIMES}

\section{A. The Ricci tensor of an $(N+1)$-dimensional metric}

Let $\hat{g}$ be a metric on an $(N+1)$-dimensional manifold $V_{N+1}$. Let us consider a foliation with a unit normal vector $n, \hat{g}(n, n)=\epsilon= \pm 1$, and let $g$ and $K$ be, respectively, the first and second fundamental forms of a slice, namely, $g=\hat{g}-\epsilon n \otimes n, K=-\frac{1}{2} \mathcal{L}_{n} g, \mathcal{L}_{n}$ being the Lie derivative with respect to $n$. For every vector field transversal to the foliation, $\xi=\alpha n+\beta, \alpha \neq 0, \hat{g}(\beta, n)=0$, there exist local coordinates $\left(x^{\mu}\right) \equiv\left(x^{0}, x^{i}\right), i=1, \ldots, N$, such that

$$
\xi=\frac{\partial}{\partial x^{0}} \equiv \partial_{0}, \hat{g}\left(n, \frac{\partial}{\partial x^{i}}\right)=0, \quad \beta=\beta^{i} \frac{\partial}{\partial x^{i}}
$$

Then the line element of the metric $\hat{g}$ may be written

$$
d \widehat{s}^{2}=\left(\epsilon \alpha^{2}+\beta^{i} \beta_{i}\right)\left(d x^{0}\right)^{2}+2 \beta_{i} d x^{i} d x^{0}+g_{i j} d x^{i} d x^{j} .
$$

Henceforth we follow the notation of [17] and all the tensorial equations will be written in covariant form. From the definition of $K$ one has

$$
\partial_{0} g=\mathcal{L}_{\beta} g-2 \alpha K
$$

The Ricci tensor of a metric $\hat{g}$ may be decomposed relatively to $n$ as $\operatorname{Ric}(\hat{g})=\sigma n \otimes n+s \otimes n+S$, where $\sigma, s$, and $S$ are, respectively, a scalar, a covariant vector, and a covariant tensor, which are orthogonal to $n$, and $\otimes$ denotes the symmetrized tensorial product, $a \otimes b=a \otimes b+b \otimes a$. From the Gauss-Codazzi relations it follows that the Ricci tensor of the metric (1) is given by

$$
\begin{aligned}
\sigma= & \operatorname{tr} K^{2}+\frac{1}{\alpha}\left(\operatorname{tr} \partial_{0} K-\operatorname{tr} \mathcal{L}_{\beta} K-\epsilon \Delta \alpha\right), \\
s= & \epsilon(d \operatorname{tr} K-\operatorname{div} K), \\
S= & \operatorname{Ric}(g)+\epsilon\left(2 K^{2}-\operatorname{tr} K K\right) \\
& +\frac{1}{\alpha}\left[\epsilon\left(\partial_{0} K-\mathcal{L}_{\beta} K\right)-D d \alpha\right],
\end{aligned}
$$

where $D$ and $\operatorname{Ric}(g)$ are, respectively, the covariant derivative and the Ricci tensor of the metric $g$ and where the following notation has been used:

$$
\begin{aligned}
& d f=\frac{\partial f}{\partial x^{i}} d x^{i}, \quad \Delta f=\operatorname{tr} D d f, \quad \operatorname{tr} K=g^{i j} K_{i j}, \\
& K^{2}=K \times K=K_{i}^{k} K_{k j} d x^{i} \otimes d x^{j}, \\
& \operatorname{div} K=g^{i j} D_{i} K_{j k} d x^{k},
\end{aligned}
$$

$f$ being any function and $K$ any tensor orthogonal to $n$.

Now we consider umbilical foliations (those for which the first and the second fundamental forms are proportional: $K=-H g$ ). Taking the vector field $\xi$ orthogonal to the slices $(\beta=0)$, from Eq. (2) it follows [18] that an $(N+1)$-dimensional space admits an umbilical foliation if and only if there exist local coordinates $\left(x^{0}, x^{i}\right)$ such that the element may be written

$$
d \widehat{s}^{2}=\epsilon \alpha^{2}\left(d x^{0}\right)^{2}+\Omega^{2} \gamma_{i j} d x^{i} d x^{j},
$$

where $\alpha=\alpha\left(x^{0}, x^{i}\right), \Omega\left(x^{0}, x^{i}\right)$, and $\gamma_{i j}=\gamma_{i j}\left(x^{k}\right)$.

Let us consider the Ricci tensor of the metric (4). Taking $\beta=0$ and $g=\Omega^{2} \gamma$, Eqs. (3) become

$$
\begin{aligned}
& \sigma=-N H^{2}-\frac{1}{\alpha}\left(N \partial_{0} H+\epsilon \Delta \alpha\right), \\
& s=\epsilon(1-N) d H, \\
& S=\operatorname{Ric}(g)-\epsilon\left(N H^{2}+\frac{1}{\alpha} \partial_{0} H\right) g-\frac{1}{\alpha} D d \alpha,
\end{aligned}
$$

where, on account of (2),

$$
H=\frac{1}{\alpha} \partial_{0} \ln \Omega .
$$

On the other hand, taking into account the relation between the connections $\nabla$ and $D$ of two conformal metrics $\gamma$ and $g=\Omega^{2} \gamma$ we have

$$
\begin{aligned}
& \frac{1}{\alpha} D d \alpha=\frac{1}{\alpha} \nabla d \alpha-d \ln \alpha \otimes d \ln \Omega+\gamma(d \ln \alpha, d \ln \Omega) \gamma, \\
& \operatorname{Ric}(g)=\operatorname{Ric}(\gamma)+(N-2) \Omega \nabla d \frac{1}{\Omega}-[\Delta \ln \Omega+(N-2) \gamma(d \ln \Omega, d \ln \Omega)] \gamma, \\
& R(g)=\Omega^{-2}\{R(\gamma)-(N-1)[2 \Delta \ln \Omega+(N-2) \gamma(d \ln \Omega, d \ln \Omega)]\},
\end{aligned}
$$

where $R($ ) is the scalar curvature of the corresponding metric. Then, as

$$
\frac{1}{f} \nabla d f=\nabla d \ln f+d \ln f \otimes d \ln f
$$

for every function $f$, substituting the two first relations (7) in (5) and considering Eq. (6), one obtains that in an ( $N+1$ )dimensional space admitting an umbilical foliation the Ricci tensor is given by 


$$
\begin{aligned}
\sigma= & -N\left(H^{2}+\frac{1}{\alpha} \partial_{0} H\right]-\frac{1}{\Omega^{2}} \epsilon[\Delta \ln \alpha+\gamma(d \ln \alpha, d \ln \alpha)+(N-2) \gamma(d \ln \alpha, d \ln \Omega)], \\
s= & \epsilon(1-N) d H, \\
S= & \operatorname{Ric}(\gamma)+(N-2)[d \ln \Omega \otimes d \ln \Omega-\nabla d \ln \Omega]-\nabla d \ln \alpha-d \ln \alpha \otimes d \ln \alpha+d \ln \alpha \otimes d \ln \Omega \\
& -\left[\Delta \ln \Omega+\epsilon \Omega^{2}\left(N H^{2}+\frac{1}{\alpha} \partial_{0} H\right]+(N-2) \gamma(d \ln \Omega, d \ln \Omega)+\gamma(d \ln \alpha, d \ln \Omega)\right] \gamma .
\end{aligned}
$$

\section{B. Einstein equations}

Let $\left(V_{3+1}, \hat{g}\right)$ be a space-time of signature +2 and $n$ a unit timelike vector. Let $\tau, t, \pi$, and $\Pi$ be, respectively, the energy density, the energy flow, the mean pressure, and the anisotropic pressures tensor, with respect to the observer $n$. Einstein equations

$$
\operatorname{Ric}(\hat{g})-\frac{1}{2} R(\hat{g}) \hat{g}=\kappa[\tau n \otimes n+t \otimes n+\pi g+\Pi]
$$

may be written

$$
\begin{aligned}
& \kappa \tau=\frac{1}{2}[\sigma+\operatorname{tr} S], \\
& \kappa t=s, \quad \kappa \Pi=S^{T}, \\
& \kappa \pi=\frac{1}{2}\left[\sigma-\frac{1}{3} \operatorname{tr} S\right],
\end{aligned}
$$

where, for any tensor $S$ orthogonal to $n$, we have written $S^{T}=S-\frac{1}{3} \operatorname{tr} S g$.

Let us assume now that the space-time admits an umbilical synchronization (foliation by spacelike hypersurfaces): there exist local coordinates $\left(x^{\mu}\right) \equiv\left(x^{0}, x^{i}\right)$, $i=1,2,3$, in which the line element is

$$
d \widehat{s}^{2}=-\alpha^{2}\left(x^{\mu}\right)\left(d x^{0}\right)^{2}+\Omega^{2}\left(x^{\mu}\right) \gamma_{i j}\left(x^{k}\right) d x^{i} d x^{j} .
$$

The Ricci tensor of the metric (10) is given by expressions (8), with $N=3$ and $\epsilon=-1$. Thus, a simple substitution into (9) leads to the following result.

Theorem 1. For the space-times that admit an umbilical synchronization, the Einstein equations may be written as follows.

Evolution equations:

$$
\begin{aligned}
\frac{1}{\alpha} \partial_{0} \ln \Omega=H, & \\
\frac{1}{\alpha} \partial_{0} H=-\frac{3}{2} H^{2}+\frac{1}{3 \Omega^{2}}( & -\frac{R(\gamma)}{4}+\Delta \ln \Omega+\Delta \ln \alpha \\
& +\gamma(d \ln \alpha, d \ln \alpha) \\
& +\gamma(d \ln \alpha, d \ln \Omega) \\
& \left.+\frac{1}{2} \gamma(d \ln \Omega, d \ln \Omega)\right]-\frac{\kappa}{2} \pi
\end{aligned}
$$

Boundary equations:

$$
\begin{aligned}
& \kappa \tau=3 H^{2}+\Omega^{-2}\left(\frac{R(\gamma)}{2}-2 \Delta \ln \Omega-\gamma(d \ln \Omega, d \ln \Omega)\right] \\
& \kappa t=2 d H, \\
& \kappa \Pi=[\operatorname{Ric}(\gamma)-\nabla d \ln \Omega-\nabla d \ln \alpha-d \ln \alpha \otimes d \ln \alpha \\
& \quad+d \ln \alpha \otimes d \ln \Omega+d \ln \Omega \otimes d \ln \Omega]^{T}
\end{aligned}
$$

Here $\nabla$ and $\Delta$ are, respectively, the covariant derivative and the Laplace operator of the initial induced metric $\gamma$.

From a kinematic point of view, an umbilical synchronization means that the unit orthogonal vector field $n=(1 / \alpha) \partial_{0}$ defines a vorticity-free and shear-free congruence. Consequently, the Petrov-Bel type of these space-times is $I, D$, or $O$ [19]. Furthermore, in terms of the canonical components of the metric (10), the expansion and the acceleration of $n$ are, respectively,

$$
\theta=3 H=\frac{3}{\alpha} \partial_{0} \ln \Omega, \quad a=d \ln \alpha .
$$

Hence, a kinematical interpretation of Theorem 1 and some interesting consequences follow.

Corollary 1. (i) If a space-time admits a vorticity-free and shear-free timelike congruence, the line element may be written as (10) and Einstein equations split into the evolution equations (11), (12) and the boundary equations (13), (14), (15), $\theta=3 H$ being the expansion, and $a=d \ln \alpha$ the acceleration of the congruence, respectively. (ii) The expansion of the congruence is homogeneous $\left[\theta=\theta\left(x^{0}\right)\right]$ if, and only if, the relative energy flow $t$ is zero. (iii) If the congruence is geodesic and with homogeneous expansion, then the relative energy flow $t$ is zero, and the relative anisotropic stress tensor $\Pi$ is invariant along the congruence; in particular, a perfect-fluid solution on an instant will be a (barotropic) perfect-fluid solution everywhere (Robertson-Walker metrics).

Let us conclude by outlining when the results of this section about space-times admitting an umbilical synchronization may be useful. In the Introduction some examples of cosmological or astrophysical interest have been quoted. These space-times also appear naturally when solving Boltzmann or Liouville equations. In fact, Treciokas and Ellis [12] have shown that a distribution function which is a solution to the Boltzmann equation may be isotropic only for a shear-free observer. On the other hand, a solution to the Liouville equation may be isotropic only for an observer defining a conformal Killing 
congruence $[4,1]$. In both cases, if the observer is vorticity-free, it will define an umbilical synchronization in the space-time.

\section{The Ricci tensor of an induced metric admitting umbilical surfaces}

We now give a result which will be used later. In Sec. IV we study the boundary equations when the induced metric $\gamma$ on the initial hypersurface admits an umbilical foliation. In this case, the Ricci tensor follows from Eq. (8), taking $N=2$ and $\epsilon=+1$.

Proposition 1. If a spacelike hypersurface $\left(V_{2+1}, \gamma\right)$ admits an umbilical foliation,

$$
\begin{array}{r}
\gamma=\mu^{2}\left(r, x^{A}\right) d r \otimes d r+Y^{2}\left(r, x^{A}\right) \Upsilon_{A B}\left(x^{C}\right) d x^{A} \otimes d x^{B}, \\
A=1,2
\end{array}
$$

its Ricci tensor $\operatorname{Ric}(\gamma)=\lambda v \otimes v+l \otimes v+L, v=\mu d r$ is given by

$$
\begin{aligned}
\lambda= & -2\left(h^{2}+\frac{1}{\mu} \partial_{r} h\right]-\frac{1}{Y^{2}}\left[\Delta_{\Upsilon} \ln \mu+\Upsilon(\mathrm{d} \ln \mu, \mathrm{d} \ln \mu)\right] \\
l= & -\mathrm{d} h \\
L= & {\left[-\nabla_{\Upsilon} \mathrm{d} \ln \mu-\mathrm{d} \ln \mu \otimes \mathrm{d} \ln \mu+\mathrm{d} \ln Y \otimes \mathrm{d} \ln \mu\right] } \\
& +\left[\frac{1}{2} R(\Upsilon)-Y^{2}\left(2 h^{2}+\frac{1}{\mu} \partial_{r} h\right]\right. \\
& \left.\quad-\Delta_{\Upsilon} \ln Y-\Upsilon(\mathrm{d} \ln \mu, \mathrm{d} \ln Y)\right] \Upsilon,
\end{aligned}
$$

where $\nabla_{\Upsilon}$ and $\Delta_{\Upsilon}$ are, respectively, the covariant derivative and the Laplace operator of the metric $\Upsilon$, and

$$
h=\frac{1}{\mu} \partial_{r} \ln Y, \quad \mathrm{~d}=\frac{\partial}{\partial x^{A}} d x^{A} .
$$

\section{SPACE-TIMES ADMITTING ISOTROPIC RADIATION}

\section{A. Summary of previous results}

For an energy tensor of the form $\hat{T}=(\rho+p) n \otimes n+p \hat{g}$ with a barotropic relation $\rho=\rho(p)$, the conservation equation is equivalent to differential relations involving the unit vector $n$ only [20]. As a particular case, one has the following result.

Lemma 1. The energy tensor $\hat{T}=(\rho+p) n \otimes n+p \hat{g}$ with $p=3 p$ is conservative if, and only if, the unit timelike vector field $n$ satisfies

$$
\widehat{d}\left(a-\frac{\theta}{3} n\right)=0
$$

$\widehat{d}$ being the exterior derivative of the space-time. Then, if $\alpha$ is such that

$$
\widehat{d} \ln \alpha=a-\frac{\theta}{3} n,
$$

the energy density and the pressure are

$$
\rho=3 p, \quad p=p_{0} \alpha^{-4},
$$

with $p_{0}$ a positive constant.

From the kinetic theory point of view, isotropic radiation is described by a distribution function $f(x, k)$, a solution of the Liouville equation along the null geodesics, and dependent on the moments $k$ only through the energy with respect to the observer $n$ measuring isotropic radiation. This function determines a macroscopic energy tensor $\hat{T}=(\rho+p) n \otimes n+p \widehat{g}$ with $\rho=3 p$. Then, on account of Lemma 1, the unitary vector field $n$ will verify Eq. (18). However, a microscopic description using the Liouville equation imposes additional conditions on the observer $n$ which lead to the existence of conformal motions on the space-time. This question was analyzed in [1] and we summarize the results below.

Lemma 2. The following statements are equivalent: (i) There exists an isotropic distribution function with respect to an observer $n, f(x, k)=h(x,-n \cdot k)$, which obeys the Liouville equation; (ii) there exists a unit timelike field $n$ such that $\sigma=0, \hat{d}\left(a-\frac{1}{3} \theta n\right)=0, \sigma, \theta$, and a being, respectively, the shear, the expansion, and the acceleration of $n$; (iii) the space-time metric is conformally stationary, that is to say, there exists a timelike field $\xi$ such that $\mathcal{L}_{\xi} \hat{g}=\Phi \hat{g}$. Moreover, the unit vector $n$ in (ii) and the conformal Killing vector in (iii) are collinear, $\xi=\alpha n, \alpha$ being a function such that $\widehat{d} \ln \alpha=a-\frac{1}{3} \theta n$; in the case of particles without mass $\left(k^{2}=0\right)$, the distribution function depends only on the first integral defined by the field $\xi$ over the null geo$\operatorname{desics}[f(x, k)=h(\xi \cdot k)]$.

\section{B. Einstein equations in the vorticity-free case}

Let us consider now space-times allowing isotropic radiation with respect to a vorticity-free observer (i.e., hypersurface orthogonal, $n \wedge \hat{d} n=0$ ). On account of Lemma 2, there exists a hypersurface orthogonal timelike conformal Killing vector; that is to say, the space-time metric is conformal to a static metric. Then, the line element may be written

$$
d \hat{s}^{2}=\Omega^{2}\left[-\Psi^{2}\left(x^{k}\right)\left(d x^{0}\right)^{2}+\gamma_{i j}\left(x^{k}\right) d x^{i} d x^{j}\right],
$$

where $\gamma$ is the initial spatial metric $\left[\Omega\left(0, x^{i}\right)=1\right]$.

On the other hand, $\alpha=\Omega \Psi$ is an integral divisor of $n$, $n=-\alpha d x^{0}$; thus, taking into account Corollary 1 , we have

$\hat{d} \ln \alpha=a-\frac{1}{\alpha}\left(\partial_{0} \ln \alpha\right) n=a-\frac{1}{\alpha}\left(\partial_{0} \ln \Omega\right) n=a-\frac{1}{3} \theta n$.

From this, and taking into account lemmas 1 and 2, we have the following result.

Proposition 2. The space-times allowing isotropic radiation with respect to a vorticity-free observer are those for which there exists an umbilical synchronization

$$
d \hat{\mathrm{s}}^{2}=-\alpha^{2}\left(x^{\mu}\right)\left(d x^{0}\right)^{2}+\Omega^{2}\left(x^{\mu}\right) \gamma_{i j}\left(x^{k}\right) d x^{i} d x^{j},
$$

such that $\alpha / \Omega$ is independent of $x^{0}$.

The observer field is $n=(1 / \alpha) \partial_{0}$, and the density and the pressure of radiation are given by $\rho=3 p, p=p_{0} \alpha^{-4}$, respectively, with $p_{0}$ a positive constant. 
Then, we may write Einstein equations for a large family of space-times admitting isotropic radiation. In fact, taking $\alpha=\Omega \Psi\left(x^{i}\right)$ in Theorem 1, we have the following.

Theorem 2. For a conformal static metric

$$
d \widehat{s}^{2}=\Omega^{2}\left[-\Psi^{2}\left(x^{k}\right)\left(d x^{0}\right)^{2}+\gamma_{i j}\left(x^{k}\right) d x^{i} d x^{j}\right],
$$

\section{Einstein equations}

$$
\begin{aligned}
\operatorname{Ric}(\hat{g})-\frac{1}{2} R(\hat{g}) \hat{g}=\kappa[\tau n \otimes n+t \otimes n+\pi g+\Pi] & \\
n & =-\Psi \Omega d x^{0}
\end{aligned}
$$

may be written.

Evolution equations:

$$
\begin{aligned}
\partial_{0} \Omega= & \Psi \Omega^{2} H, \\
\partial_{0} H= & -\frac{3}{2} \Psi \Omega H^{2} \\
& +\frac{\Psi}{3 \Omega}\left(-\frac{R(\gamma)}{4}+\Delta \ln \Psi+\gamma(d \ln \Psi, d \ln \Psi)\right. \\
& +2 \Delta \ln \Omega+\frac{5}{2} \gamma(d \ln \Omega, d \ln \Omega) \\
& +3 \gamma(d \ln \Psi, d \ln \Omega)]-\frac{\kappa}{2} \Omega \Psi \pi .
\end{aligned}
$$

Boundary equations:

$$
\begin{aligned}
& \kappa \tau=3 H^{2}+\Omega^{-2}\left(\frac{R(\gamma)}{2}-2 \Delta \ln \Omega-\gamma(d \ln \Omega, d \ln \Omega)\right] \\
& \kappa t=2 d H, \\
& \kappa \Pi=[\operatorname{Ric}(\gamma)-\nabla d \ln \Psi-d \ln \Psi \otimes d \ln \Psi \\
& \quad-2 \nabla d \ln \Omega+2 d \ln \Omega \otimes d \ln \Omega]^{T}
\end{aligned}
$$

Here $\nabla$ and $\Delta$ are, respectively, the covariant derivative and the Laplace operator of $\gamma$.

Theorem 2 gives the general expression of Einstein equations in the $3+1$ way for space-times allowing isotropic radiation with respect to a hypersurface orthogonal observer. If we want solutions modeling space-times with physical properties established beforehand, we have to impose conditions restricting the field variables. For instance, when the energy flow $t$ is zero, the expansion $H$ is homogeneous; the nature of anisotropic pressures II will affect the spatial metric $\gamma$ and the spatial dependence of the functions $\Psi$ and $\Omega$; an hypothesis about the mean pressure $\pi$ will have an influence on the evolution of the metric.

The important result of Elhers, Geren, and Sachs [1] quoted in the Introduction establishing a relation between the isotropy of MWB and Robertson-Walker universes can now be obtained easily. In fact, when the observer $n$ who sees isotropic radiation is geodesic and expanding, from (19) it follows that $H=H\left(x^{0}\right) \neq 0$, and (18) implies that $n$ is vorticity-free. Thus the hypothesis of Corollary 1 (iii) is satisfied and we have the following.
Corollary 2. Any space-time that admits an expanding and geodesic observer measuring isotropic radiation (solution to Liouville equation), and that is a perfect fluid on an instant, is a Robertson-Walker universe.

\section{SOLUTIONS WITH ANISOTROPIC PRESSURE IN THE ENERGY FLOW DIRECTION}

In this section we will study the Einstein equations for conformal static space-times when in the energy flow direction $t$ there exists a pressure $\pi_{r}$ which is different from the transversal pressures $\pi_{\perp}$ in the orthogonal twoplane. That is to say, we consider an energy tensor

$\widehat{T}=\tau n \otimes n+|t| e \otimes n+\pi_{r} e \otimes e+\pi_{1}(g-e \otimes e), \quad \widehat{g}(e, e)=1$.

In this case, it is possible to substitute the mean pressure $\pi$ and the anisotropic pressures $\Pi$ by the radial and transverse pressures $\pi_{r}$ and $\pi_{\perp}$ :

$$
3 \pi=\pi_{r}+2 \pi_{\perp}, \quad \Pi=\left(\pi_{r}-\pi_{\perp}\right)\left(e \otimes e-\frac{1}{3} g\right) .
$$

Thus, in the evolution equation (20) the freedom in the mean pressure $\pi$ is replaced by a free election of the transverse pressure $\pi_{1}$, and (23) becomes a unique boundary equation.

In order to obtain solutions with an energy tensor of type (24), we need to impose additional conditions to the metric. Let us consider two cases.

\section{Case I}

One requires that functions $\Omega$ and $\Psi$ depend on a unique spatial coordinate $r$ :

$$
\Omega=\Omega\left(x^{0}, r\right), \quad \Psi=\Psi(r) .
$$

Moreover one states that on the initial hypersurface $x^{0}=0$ the surfaces $r=$ const are umbilical with geodesic normal and homothetic induced metrics. That is to say, the initial spatial metric $\gamma$ may be written

$\gamma=d r \otimes d r+Y^{2}(r) \Upsilon_{A B}\left(x^{C}\right) d x^{A} \otimes d x^{B}, \quad A=1,2$.

Conditions (26) and (27) lead to

$d \ln \Psi=(\ln \Psi)^{\prime} d r, \quad \nabla d \ln \Psi=(\ln \Psi)^{\prime \prime} d r \otimes d r+(\ln \Psi)^{\prime} \nabla d r$,

$d \ln \Omega=B d r, \quad \nabla d \ln \Omega=\partial_{r} B d r \otimes d r+B \nabla d r$,

$B=\partial_{r}(\ln \Omega), \quad \nabla d r=h(\gamma-d r \otimes d r), \quad h=(\ln Y)^{\prime}$,

where, for a function $f(r), f^{\prime}=d f / d r$. Then, taking into account Proposition 1 with $\mu=1$ and $Y\left(r, x^{A}\right)=Y(r)$, the Ricci tensor of the metric (27) is

$$
\begin{aligned}
\operatorname{Ric}(\gamma)= & \frac{2}{3}\left(-3 h^{2}-2 h^{\prime}+\frac{G(\Upsilon)}{Y^{2}}\right) \gamma \\
& -\left(h^{\prime}+\frac{G(\Upsilon)}{Y^{2}}\right)\left(d r \otimes d r-\frac{1}{3} \gamma\right),
\end{aligned}
$$

$G(\Upsilon)$ being the Gauss curvature of the metric $\Upsilon$, $G(\Upsilon)=2 \operatorname{tr} \operatorname{Ric}(\Upsilon)$. Relative to $n$, the magnetic part of 
the Weyl tensor vanishes, and its electric part has two equal eigenvalues; thus, its Petrov-Bel type is $D$ or $O$. The Einstein equations follow from Theorem 2. In fact, substituting (28) and (29) in Eqs. (19) - (23), taking into account relations $(25)$ and introducing $B$ as an evolution variable, we conclude that the Einstein equations for the metric

$$
\begin{aligned}
d \hat{s}^{2}=\Omega^{2}\left(x^{0}, r\right)[ & -\Psi^{2}(r)\left(d x^{0}\right)^{2}+(d r)^{2} \\
& \left.+Y^{2}(r) \Upsilon_{A B}\left(x^{C}\right) d x^{A} d x^{B}\right]
\end{aligned}
$$

may be written as follows.

Evolution equations:

$$
\begin{aligned}
\partial_{0} \Omega=\Psi \Omega^{2} H & \\
\partial_{0} H=-\frac{3}{2} \Psi \Omega H^{2}+\frac{\Psi}{\Omega} & {\left[c_{0}+\frac{1}{2} B^{2}+\partial_{r} B\right.} \\
& \left.+B\left[\frac{Y^{\prime}}{Y}+\frac{\Psi^{\prime}}{\Psi}\right)\right]-\frac{\kappa}{2} \Omega \Psi \pi_{1}
\end{aligned}
$$

Boundary equations:

$$
\begin{aligned}
& \kappa \tau=3 H^{2}+\Omega^{-2}\left(\eta_{0}-2 \partial_{r} B-4 B \frac{Y^{\prime}}{Y}-B^{2}\right), \\
& \kappa|t|=\frac{2}{\Omega} \partial_{r} H, \\
& \kappa\left(\pi_{r}-\pi_{\perp}\right)=\Omega^{-2}\left[b_{0}+2\left[B \frac{Y^{\prime}}{Y}-\partial_{r} B+B^{2}\right]\right] .
\end{aligned}
$$

Here

$$
\begin{aligned}
& c_{0}=\frac{1}{2 \Psi Y}\left(Y^{\prime \prime} \Psi+Y \Psi^{\prime \prime}+Y^{\prime} \Psi^{\prime}\right), \\
& b_{0}=\chi_{0}+\frac{Y^{\prime} \Psi^{\prime}}{Y \Psi}-\frac{\Psi^{\prime \prime}}{\Psi}, \\
& \eta_{0}=\frac{G(\Upsilon)}{Y^{2}}-\frac{Y^{\prime 2}}{Y^{2}}-2 \frac{Y^{\prime \prime}}{Y}, \\
& \chi_{0}=-\frac{G(\Upsilon)}{Y^{2}}+\frac{Y^{\prime 2}}{Y^{2}}-\frac{Y^{\prime \prime}}{Y} .
\end{aligned}
$$

The evolution Eqs. (31)-(33) have the form

$$
\partial_{0} U=\mathcal{A} \partial_{r} U+\mathcal{F}(U)
$$

with

$$
U \equiv(\Omega, H, B), \quad \mathcal{A}=\left(\begin{array}{ccc}
0 & 0 & 0 \\
0 & 0 & \Psi / \Omega \\
0 & \Psi \Omega & 0
\end{array}\right] .
$$

The matrix $\mathcal{A}$ has eigenvalues $0, \Psi$ and $-\Psi$; that is to say, (41) is a strictly hyperbolic system. This is a suitable property when one looks for numerical solutions.

In order to obtain particular solutions for a metric of the form (30), it may be suitable to proceed in three steps.

(1) Resolution of the boundary Eqs. (34)-(36) on the initial hypersurface $x^{0}=0$ :

Equations: $\kappa \tau_{0}=3 H_{0}^{2}+\eta_{0}$,

$$
\begin{aligned}
& \kappa\left|t_{0}\right|=2 H_{0}^{\prime}, \\
& \kappa\left(\pi_{r}-\pi_{\perp}\right)_{0}=b_{0} .
\end{aligned}
$$

Sources: $\left(\tau_{0},\left|t_{0}\right|,\left(\pi_{r}-\pi_{\perp}\right)_{0}\right)$.

Unknown quantities: $\left(Y(r), \Psi(r), H_{0}(r), \Upsilon\left(x^{A}\right)\right)$.

(2) Resolution of the evolution equations once one has substituted the functions $Y$ and $\Psi$ already obtained:

Equations: (31), (32), (33) .

Sources: $\pi_{1}$.

Unknown quantities: $U \equiv(\Omega, H, B)$,

$$
U(0, r)=\left(1, H_{0}, 0\right) \text {. }
$$

(3) Determination of the space-time energy distributions:

Total energy tensor: $\hat{T}=\tau n \otimes n+|t| e \otimes n+\pi_{r} e \otimes e$

$$
+\pi_{\perp}(g-e \otimes e) \text {, }
$$

with $e=\Omega d r$, and where $\tau,|t|$, and $\pi_{r}-\pi_{\perp}$ are given, respectively, by (34), (35), and (36).

Radiation energy distribution:

$$
\rho_{\mathrm{rad}}=3 p_{\mathrm{rad}}, \quad p_{\mathrm{rad}}=\frac{p_{0}}{\Psi^{4} \Omega^{4}} .
$$

\section{Case II}

Let us consider now the class of conformal static space-times whose line element is of the form

$d \hat{s}^{2}=\Omega^{2}\left(x^{0}\right)\left(-\Psi^{2}\left(x^{k}\right)\left(d x^{0}\right)^{2}+\frac{1}{\Psi^{2}\left(x^{k}\right)} c_{i j}\left(x^{k}\right) d x^{i} d x^{j}\right)$,

$c$ being a metric of constant curvature, $\operatorname{Ric}(c)=\frac{2}{3} \eta_{0} c$.

From Theorem 2, taking $\Omega=\Omega\left(x^{0}\right)$ and $\gamma=(1 / \Psi) c$, and taking into account relations (25), the Einstein equations for the metric (45) may be written

$$
\begin{aligned}
& \kappa \tau=\frac{1}{\Omega^{2}}\left[\frac{3 \dot{\Omega}^{2}}{\Psi^{2} \Omega^{2}}+2 \Psi \Delta \Psi-3 c(d \Psi, d \Psi)+\Psi^{2} \eta_{0}\right], \\
& \kappa t=-\frac{2 \dot{\Omega}}{\Psi^{2} \Omega^{2}} d \Psi, \\
& \kappa \pi_{r}=\frac{1}{\Omega^{2}}\left[\frac{1}{\Psi^{2}}\left[\frac{\dot{\Omega}^{2}}{\Omega^{2}}-\frac{2 \ddot{\Omega}}{\Omega}\right]-c(d \Psi, d \Psi)-\frac{1}{3} \Psi^{2} \eta_{0}\right],
\end{aligned}
$$


$\kappa \pi_{\perp}=\frac{1}{\Omega^{2}}\left[\frac{1}{\Psi^{2}}\left[\frac{\dot{\Omega}^{2}}{\Omega^{2}}-\frac{2 \ddot{\Omega}}{\Omega}\right]+c(d \Psi, d \Psi)-\frac{1}{3} \Psi^{2} \eta_{0}\right]$,

where $\nabla$ and $\Delta$ are, respectively, the covariant derivative and the Laplacian operator of the metric $c$, and $\dot{\Omega}=d \Omega / d x^{0}$.

The metrics (45) become the Robertson-Walker metrics when $\Psi=1$. Thus taking $\Psi=1+\phi$ with $\phi<<1$, we can study perturbed Robertson-Walker universes which admit isotropic radiation.

\section{CONCLUSIONS}

The main results of this paper are summarized in two theorems: we have written Einstein equations in the $3+1$ way for space-times with a vorticity-free and shear-free observer (Theorem 1), and for space-times admitting isotropic radiation with respect to a vorticity-free observer
(Theorem 2). As Corollary 2 illustrates, if we want space-times compatible with isotropic radiation other than Robertson-Walker universes, we must allow for a nongeodesic observer or for sources other than a comoving perfect fluid.

In Sec. IV we have considered a special type of anisotropic pressure that we think may have physically interesting applications. In the first case, the evolution equations become a strictly hyperbolic system and the numerical methods may be applied. Elsewhere [21] we have showed that in this case an evolution governed by an ordinary second-order differential equation is possible, and we have constructed a model of spherical cluster. A second case includes a family of perturbed Friedmann models compatible with an isotropic distribution of photons.

\section{ACKNOWLEDGMENTS}

The authors acknowledge the financial support of the Spanish DGICyT, project No. PB90-0416.
[1] J. Ehlers, P. Geren, and R. K. Sachs, J. Math. Phys. 9, 1344 (1968).

[2] S. W. Hawking and G. F. R. Ellis, The Large Scale Structure of Space-Time (Cambridge University Press, Cambridge, England, 1973).

[3] R. K. Sachs and A. M. Wolfe, Astrophys. J. 147, 73 (1967).

[4] G. E. Tauber and J. W. Weinberg, Phys Rev. 122, 1342 (1961).

[5] J. J. Ferrando, J. A. Morales, and M. Portilla (unpublished).

[6] H. Stephani, Commun. Math. Phys. 4, 137 (1967).

[7] C. Bona, in Proceedings of the Spanish Relativistic Meeting, Santander, Spain, 1984, edited by M. Portilla, J. L. Sanz, and R. Lapiedra (Universitat de València, València, Spain, 1985).

[8] B. Coll and J. A. Morales, J. Math. Phys. 32, 2450 (1991).

[9] H. Takeno, The Theory of Spherically Symmetric SpaceTimes, Science Reports of the Research Institute of Theoretical Physics No. 5 (Hiroshima University, Hiroshi- ma, Japan, 1966).

[10] C. Bona and B. Coll, Gen. Relativ. Gravit. 20, 293 (1988).

[11] J. J. Ferrando, J. A. Morales, and M. Portilla (in preparation).

[12] R. Treciokas and G. F. R. Ellis, Commun. Math. Phys. 23, 1 (1974).

[13] J. J. Ferrando, J. A. Morales, and M. Portilla, Phys. Rev. D 40, 1027 (1989).

[14] A. M. Fridman and V. L. Polyachenco, Physics of Gravitating Systems (Springer-Verlag, New York, 1984).

[15] D. Merrit, Astrophys. J. 313, 121 (1987).

[16] J. J. Ferrando, J. A. Morales, and M. Portilla, Gen. Relativ. Gravit. 22, 1021 (1990).

[17] B. Coll, Thèse d'état, Paris, 1980.

[18] L. P. Eisenhart, Riemannian Geometry (Princeton University Press, Princeton, NJ, 1949).

[19] M. Trümper, J. Math. Phys. 6, 585 (1965).

[20] B. Coll and J. J. Ferrando, J. Math. Phys. 31, 1020 (1990).

[21] J. J. Ferrando, J. A. Morales, and M. Portilla (in preparation). 